\title{
Erratum to: High precision radial velocities with GIANO spectra
}

\author{
I. Carleo ${ }^{1,2} \cdot$ N. Sanna ${ }^{3} \cdot$ R. Gratton ${ }^{1}$. \\ S. Benatti ${ }^{1}$ - M. Bonavita ${ }^{4}$ - E. Oliva ${ }^{3} \cdot$ L. Origlia $^{5}$. \\ S. Desidera ${ }^{1} \cdot$ R. Claudi $^{1} \cdot$ E. Sissa ${ }^{1}$
}

Published online: 23 July 2016

(C) Springer Science+Business Media Dordrecht 2016

\section{Erratum to: Exp Astron \\ DOI: 10.1007/s10686-016-9492-3}

The original publication contained an error. Table 6 Number of stars accessible to GIANO/TNG for a given RV precision, the RV precision is in $\mathrm{m} \mathrm{s}^{-1}$ and not in $\mathrm{km} \mathrm{s}^{-1}$. The corrected table is reproduced here.

Table 6 Number of stars accessible to GIANO/TNG for a given RV precision (data based on the 2MASS all-sky catalog of point Sources [10])

\begin{tabular}{lll}
\hline H magnitude & $\begin{array}{l}\text { RV precision } \\
\left(\mathrm{m} \mathrm{s}^{-1}\right)\end{array}$ & $\begin{array}{l}\text { Number of stars } \\
\left(10^{4}\right)\end{array}$ \\
\hline 5.00 & 10 & 3 \\
5.75 & 20 & 4.6 \\
6.50 & 40 & 5.6 \\
7.50 & 100 & 15 \\
\hline
\end{tabular}

The online version of the original article can be found at http://dx.doi.org/10.1007/s10686-016-9492-3.

I. Carleo

ilaria.carleo@oapd.inaf.it

1 INAF - Astronomical Observatory of Padua, Vicolo dell'Osservatorio 5, 35122 Padova, Italy

2 Department of Physics and Astronomy "Galileo Galilei”, University of Padua, Via Marzolo, 8, 35131 Padova, Italy

3 INAF - Astronomical Observatory of Arcetri, Largo Enrico Fermi, 5, 50125 Florence, Italy

4 Institute for Astronomy, The University of Edinburgh, Royal Observatory, Blackford Hill, Edinburgh EH9 3HJ, UK

5 INAF - Astronomical Observatory of Bologna, Via Ranzani, 1, 40127 Bologna, Italy 